\title{
Phase Transitions Governed by the Fifth Power of the Golden Mean and Beyond
}

\author{
Hans Hermann Otto \\ Materials Science and Crystallography, Clausthal University of Technology, Clausthal-Zellerfeld, Lower Saxony, Germany \\ Email: hhermann.otto@web.de
}

How to cite this paper: Otto, H.H. (2020) Phase Transitions Governed by the Fifth Power of the Golden Mean and Beyond. World Journal of Condensed Matter Physics, 10, 135-158.

https://doi.org/10.4236/wjcmp.2020.103009

Received: June 29, 2020

Accepted: August 3, 2020

Published: August 6, 2020

Copyright (c) 2020 by author(s) and Scientific Research Publishing Inc. This work is licensed under the Creative Commons Attribution International License (CC BY 4.0).

http://creativecommons.org/licenses/by/4.0/ (c) (i) Open Access

\begin{abstract}
In this contribution results from different disciplines of science were compared to show their intimate interweaving with each other having in common the golden ratio $\varphi$ respectively its fifth power $\varphi^{5}$. The research fields cover model calculations of statistical physics associated with phase transitions, the quantum probability of two particles, new physics of everything suggested by the information relativity theory (IRT) including explanations of cosmological relevance, the $\varepsilon$-infinity theory, superconductivity, and the Tammes problem of the largest diameter of $N$ non-overlapping circles on the surface of a sphere with its connection to viral morphology and crystallography. Finally, Fibonacci anyons proposed for topological quantum computation (TQC) were briefly described in comparison to the recently formulated reverse Fibonacci approach using the Janičko number sequence. An architecture applicable for a quantum computer is proposed consisting of 13-step twisted microtubules similar to tubulin microtubules of living matter. Most topics point to the omnipresence of the golden mean as the numerical dominator of our world.
\end{abstract}

\section{Keywords}

Golden Mean, Phase Transitions, Hard-Hexagon Respectively Hard-Square Gas Model, Quantum Probability, Information Relativity Theory (IRT), $\mathcal{\varepsilon}$-Infinity Theory, Superconductivity, Tammes Problem, Viral Morphology, Helical Microtubules, Janičko Number Sequence, Topological Quantum Computation, Fibonacci Lattice, Crystallography

\section{Introduction}

The golden mean or golden ratio is an omnipresent number in nature, found in the architecture of living creatures as well as human buildings, music, finance, medicine, philosophy, and of course in physics and mathematics including quan- 
tum computation [1] [2] [3]. It is the most irrational number known with the simplest continued fraction representation at all [4] and a number-theoretical chameleon with a self-similarity property. All these properties render it to be suitable for quantum computer application. According to Olson et al. [2], the whole universe functions as a golden supercomputer. The seminal $\mathcal{E}$-infinity approach developed by El Naschie years ago and applied to cosmological questions based on this simple principle of nature [5]. El Naschie and his scientific fellows condensed these ideas to a grand unification of the sciences, arts and consciousness [2]. Before also the great women scientists Mae-Wan Ho [6] and Leila Marek-Crnjac [2] [7] made valuable contributions to this topic as well as Ji-Huan $\mathrm{He}$ [2]. Another contribution of Klee Irwin about unification of physics and number theory takes a similar approach using statements from quantum physics [8].

However, recently the unification of physics was advanced by Suleiman's information relativity theory (IRT) correcting an overlooked flaw in Newton's theory with respect to time displacements between observer and moving bodies [9]. Many formal physical constructs are overcome by this new insight.

Whereas the unification of physics is intimately connected with the golden number system, in this contribution the main focus is simply on phase transformation governed by the fifth power of the golden mean and insights beyond this topic.

Herein the golden mean is denoted by $\varphi$. Its inverse number is just $\varphi^{-1}=1+\varphi$. One yields numerically

$$
\begin{gathered}
\varphi=\frac{\sqrt{5}-1}{2}=0.6180339887 \ldots \\
\varphi^{-1}=\frac{\sqrt{5}+1}{2}=1+\varphi=1.6180339887 \ldots
\end{gathered}
$$

It is important to notice that in numerous papers, for instance such about topological quantum computation $(T Q C)$, the golden mean is taken as $\frac{\sqrt{5}+1}{2}$. However, in emerging matrix representations for Fibonacci anyon models actually the inverse of this number is always found in the matrix elements. So the choice of the golden ratio according to Equation (1) is well founded and should be maintained.

While in nature this number emerges as result of chaotic-statistical evolution, on the other hand, the fifth power of $\varphi$ is frequently linked to phase transitions from microscopic to cosmic scale. The secrete behind both number may be revealed by considering their infinitely continued fraction representations [4] indicating how simple nature's most effective evolutionary forecast works

$$
\varphi=\frac{\sqrt{5}-1}{2}=\frac{1}{1+\frac{1}{1+\frac{1}{1+\cdots}}}
$$




$$
\varphi^{5}=\frac{1}{11+\frac{1}{11+\frac{1}{11+\cdots}}}
$$

Rogers-Ramanujan presented a continued fraction of the form [4] [10]

$$
r(\tau)=\frac{q^{1 / 5}}{1+\frac{q}{1+\frac{q^{2}}{1+\frac{q^{3}}{1+\cdots}}}}
$$

where $q=\mathrm{e}^{2 \pi i \tau}$. For $\tau=0$ one gets the golden mean $\varphi$, and for $\tau=i=\sqrt{-1}$ the interesting result is [4]

$$
\begin{aligned}
r(i) & =\sqrt{\frac{5+\sqrt{5}}{2}}-\frac{1+\sqrt{5}}{2}=\sqrt{2+\varphi^{-1}}-\varphi^{-1} \\
& \approx \pi \varphi^{5}+\left(\frac{\pi \varphi^{5}}{10}\right)^{2}
\end{aligned}
$$

which can be approximated by terms containing $\varphi^{5}$. About a nested infinitely continued fraction calculating $\tilde{\varphi}=0.619071 \cdots$ see [4]. Due to the property of the golden number its fifth power can be traced back to the simple relation $\varphi^{5}=5 \varphi-3 \quad$ (see also Appendix).

However, if one deals especially with the behavior of matter suffering from phase transitions, then the ideas of Baxter caused the deepest impact on physics [11] [12] [13] and will be reviewed in some detail in the following chapter. Then Hardy's quantum probability function of two particles was investigated in more detail and connected with results of mathematical statistics and statistical mechanics [14] [15]. Surprisingly, Hardy's function is found in the matter energy density relation resulting from Suleiman's information relativity theory (IRT), too [9] [16]. The maximum of this energy density relation at a recession velocity of $\varphi$ yielded exactly $\varphi^{5}$ and was attributed to criticality of a transition at cosmic scale [9]. Another phase transition of economic relevance, the superconducting transition, also indicates a $\varphi^{5}$ relation [17] [18] [19]. After this a further topic is included, namely Tammes problem of the largest diameter of $N$ non-overlapping circles on the surface of a sphere with its connection to viral morphology and crystallography [20]. Finally, Fibonacci anyons proposed for topological quantum computation (TQC) were briefly described [21] [22] in comparison to the recently formulated reverse Fibonacci approach using the Janicko number sequence [23]. All these ideas are pioneering for applications including, besides mathematics and geometry, cosmology, crystallography, electrostatics, music, botany, viral morphology as well as quantum computation in connection with human mind and consciousness, and point to the omnipresence of the golden mean as the numerical dominator of our world.

It remains to be noted that it was a long way before Baxters ideas were fully accepted by down-to-earth physicists, and it is hoped that the new ideas formu- 
lated by Suleiman respectively El Naschie and scientific fellows will be soon taken over by the scientific community.

\section{Phase Transition in the Hard Hexagon Lattice Gas Model}

Simplistic models of statistical mechanics covering a large system of particles are important in linking microscopic and macroscopic average properties and behavior of matter undergoing a transition from disordered states to ordered ones by density increase or temperature reduction. The Ising model of a ferromagnet was the first exactly solved two-dimensional model defined on a square lattice [24].

In the following we want to pay tribute to Baxter's important research in the field of statistical mechanics [11] [12] [13], dealing with the geometry and phase transition of a hard two-dimensional hexagonal lattice gas model, where hard means non-overlapping hexagons. Figure 1 illustrates a possible distribution of such non-overlapping hexagons in a triangular lattice cutout. A synopsis of results besides selected references for models of rigid gas molecules on a regular lattice is given by Finch [25]. Baxter was able to give an almost exact solution of this hard-hexagon model. For such a model the grand-canonical partition function $Z$ is given by

$$
Z(z)=\sum_{n=0}^{\frac{N}{3}} z^{n} g(n, N)
$$

where the positive real variable $z$ denotes activity or fugacity, and $g(n, N)$ defines the number of allowed ways of placing $n$ particles (molecules) on the lattice. One can write the partition function per site of an infinite lattice as

$$
\kappa=\lim _{n \rightarrow \infty} Z^{1 / N}
$$

Low activity is equivalent to homogeneity, whereas high activity complies with heterogeneity. In this way, a phase transition is expected. Baxter discovered two distinct regions of positive fugacity with criticality at the point $z=Z_{c}$

$$
0 \leq z<z_{c} \text { and } z_{c}<z<\infty
$$

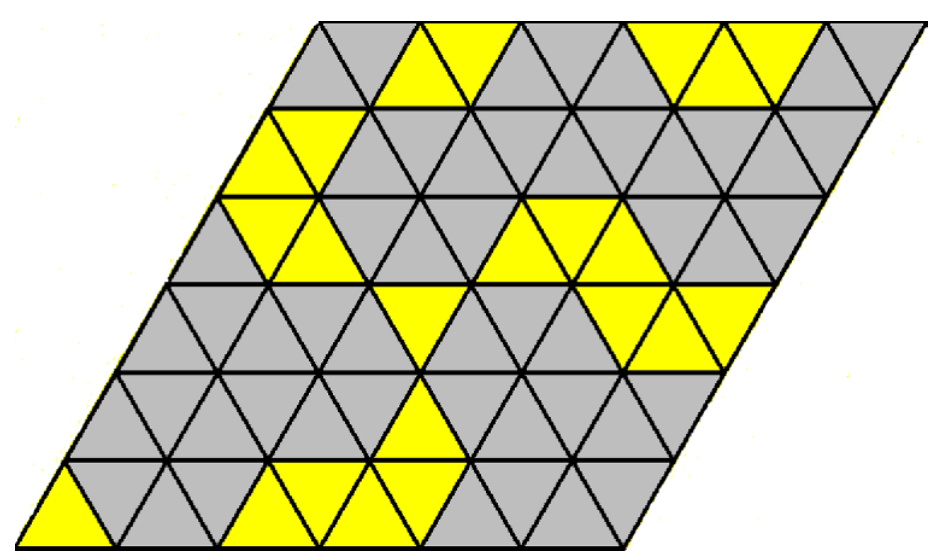

Figure 1. Statistical sites of hard (non-overlapping) hexagons in a triangular lattice cutout. 
Quite surprisingly, the result for the critical $Z_{c}$ is numerically equal to the reciprocal fifth power of the golden ratio

$$
z_{c}=\varphi^{-5}=11+\varphi^{5}=11.0901699 \cdots
$$

A second (non-physical) singularity was found for $z_{n p}=-\varphi^{5}=-0.090169943 \cdots$ The two singularities are the roots of the simple quadratic equation

$$
z_{c}^{2}-a z_{c}-1=0
$$

where $a=11$. For $a=-1$ one simply gets the golden mean $\varphi=\frac{\sqrt{5}-1}{2}$ as one of the solutions. So we are once again faced with the importance of the golden mean in statistical mechanics.

The behavior of the mean density $\rho(z)$ and the order parameter $R(z)$ at the critical point has been calculated, too. By using the normalized value $Z_{r}=\frac{Z}{Z_{c}}$ one confirms

$$
\rho(z) \sim \rho_{c}-5^{-\frac{3}{2}}\left(1-z_{r}\right)^{\frac{2}{3}}=\rho_{c}-\frac{1}{\varphi^{5}+\varphi^{-5}}\left(1-z_{r}\right)^{\frac{2}{3}}, z \rightarrow z_{c}^{-} \quad \text { (low density branch) }
$$

where

$$
\rho_{c}=\frac{5-\sqrt{5}}{10}=\frac{\varphi}{\sqrt{5}}=0.276393202 \cdots
$$

$R(z)=\frac{3}{\sqrt{5}}\left[\frac{1}{5 \sqrt{5}}\left(z_{r}-1\right)\right]^{\frac{1}{9}}=\frac{3}{\sqrt{5}}\left[\frac{1}{\varphi^{5}+\varphi^{-5}}\left(z_{r}-1\right)\right]^{\frac{1}{9}}, z \rightarrow z_{c}^{+} \quad$ (high density branch)

It is worth to note that the critical exponents $2 / 3$ and $1 / 9$ are conjectured to be universal. Further one should notice that the pre-factors given in the original publication are simply the reciprocal of the sum of the fifth power of the golden mean and its inverse

$$
5^{-\frac{3}{2}}=\frac{1}{5 \sqrt{5}}=\frac{1}{\varphi^{5}+\varphi^{-5}}=11+2 \varphi^{5} .
$$

For a more precise study of the hard hexagon partition function in the complex fugacity plane see the valuable contribution published by Assis et al. [26].

Following Tracy et al. [27], the fugacity $Z(\tau)$ can be derived from Klein's icosahedron function $\varsigma(\tau)$ [27] [28] as a branched cover (analytic bijection) of the Riemann sphere giving

$$
Z(\tau)=\left\{\begin{array}{lc}
-\varsigma^{5}(\tau) & \text { disordered regime } \\
\varsigma^{-5}(\tau) & \text { ordered regime }
\end{array}\right.
$$

which is compatible with Baxters results for the hard hexagon approach [11] [12] [13]. The close connection between an icosahedron as a regular solid and the theory of groups should be noted here. Re-expressing the approach of Assis et al. [26] by likewise using the complex variable $z=\varsigma^{5}$, the golden ratio $\varphi$ and the fifth root of unity $\omega_{1}$ (see Appendix). 


$$
\omega_{1}=\frac{\varphi}{2}+\frac{i}{2} \sqrt{1+\varphi^{-2}},\left|\omega_{1}\right|=1,
$$

and applying the $h_{5}$ order-5 transformation

$$
\varsigma \rightarrow h_{5}(\varsigma)=\frac{\omega_{1}-\varphi \varsigma}{\omega_{1} \varphi+\varsigma}
$$

then due to this transformation the Hauptmodul $H(\varsigma)$ is invariant by the involution $\varsigma \rightarrow-\varsigma^{-1}$ and as well by the order-5 transformation $\varsigma \rightarrow \omega_{1} \cdot \varsigma$

$$
H(\varsigma)=H\left(h_{5}(\varsigma)\right)=H\left(-\varsigma^{-1}\right)=H\left(\omega_{1} \cdot \varsigma\right)
$$

\section{Hard Square Lattice Model}

It was also Baxter [12] [13] who introduced the two-dimensional hard-square lattice model. Figure 2 depicts a statistical site occupation cutout based on an illustration given by Finch [25].

Baxter showed that this model is integrable resulting in two critical points at fugacities $Z_{c}$

$$
z_{c}= \pm \sqrt{\varphi^{5}}
$$

Again the fifth power of the golden mean is involved in the result. Baxter's famous work was seemingly overlooked and therefore not quoted in the Science article about quantum criticality, which was experimentally verified in the golden ratio bearing spin dynamics next a phase critical point of the quasi-onedimensional Ising ferromagnet $\mathrm{CoNb}_{2} \mathrm{O}_{8}$ published by Coldea et al. [29].

Beyond it should be mentioned that the quantum Hamiltonian of a one-dimensional hard-boson model was found to map the transfer matrix of Baxter's hard-square model with a quantum critical point at [30]

$$
\frac{V}{w}=\sqrt{\varphi^{5}}
$$

where $V$ represents the nearest-neighbor (two sites apart) interaction, $U$ the chemical potential of the bosons, and $w^{2}=V^{2}+U V$. The Hilbert space of the chosen one-dimensional quantum approach is shown to be identical of the space of states along a line of the hard-square model [30].

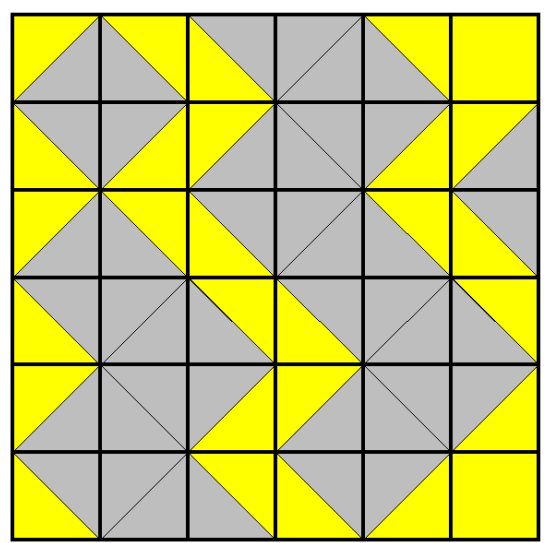

Figure 2. Hard squares sites on the square lattice [25]. 


\section{Results of $\varepsilon$-Infinity Theory and Beyond}

El Naschie's E-infinity $\left(\varepsilon^{\infty}\right)$ theory [5] originates from a fractal Cantorian set theory [31] as a number-theoretical route of physics for explaining the dualism between particles and waves that can serve solving cosmological mysteries such as dark matter and dark energy [5]. The approach delivers effective quantum gravity formulas for the cosmological mass (energy) constituents of baryonic matter $e_{M}$, dark matter $e_{D M}$, entire dark constituents $e_{E D}$, and pure dark energy $e_{P D}$ as follows

$$
\begin{gathered}
e_{M}=\frac{\varphi^{5}}{2}=0.04508497 \\
e_{E D}=1-e_{M}=\frac{5}{2} \varphi^{2}=0.9549150 \\
e_{D M}=\frac{3}{2} \varphi^{4}=0.218847 \\
e_{P D}=2 \varphi-\frac{1}{2}=0.736068 \\
e_{M}+e_{D M}+e_{P D}=1
\end{gathered}
$$

The readers are faced in the resulting formulas with the golden mean, especially with its fifth power in the beautiful relation

$$
e_{M}+e_{E D}=\frac{\varphi^{5}}{2}+\frac{5}{2} \varphi^{2}=1
$$

where matter is completed by its dark wavy surrounding.

In a previously published contribution the present author recast both the matter amount $e_{M}$ and dark matter one $e_{D M}$ into a suitable form by combining $\varphi^{5}$ and the inverse number to suggest a reciprocity relation between both matter entities according to [17] [19]

$$
\begin{gathered}
e_{M}=\frac{1}{10} 5 \varphi^{5}=0.04508 \cdots \\
e_{D M}=\frac{1}{10}\left(5 \varphi^{5}\right)^{-1}=0.22180 \cdots
\end{gathered}
$$

Then a persuasive equation for the pure dark energy $e_{P D}$ can be written down [17] [19]

$$
e_{P D}=1-\frac{1}{10}\left(5 \varphi^{5}+\left(5 \varphi^{5}\right)^{-1}\right)=0.7331(73.31 \%)
$$

Such quantum entanglement based coincidence means that the constituents of the cosmos should not be considered independent of each other, which was impressively confirmed later by the IRT theory of Suleiman [9].

\section{Hardy's Quantum Probability and Suleiman's Matter Energy Density Relation}

Hardy's maximum quantum probability of two quantum particles [14] [15] ex- 
actly equals the fifth power of $\varphi$ (Figure 1 ). This asymmetric probability distribution function with $p_{\tau}$ as entanglement variable, running from not entangled states to completely entangled ones, is given by

$$
P=p_{\tau}^{2} \frac{1-p_{\tau}}{1+p_{\tau}}
$$

The maximum of $P$ yielded

$$
P_{\max }=\frac{1-\varphi}{1+\varphi} \varphi^{2}=\varphi^{5}=0.090169943 \cdots
$$

The Hardy function, displayed in Figure 3, turns out to be a central topic of the scale-free Information Relativity theory (IRT) of Suleiman [9] by mapping the transformation of his relative matter energy density. Suleiman characterized the behavior at the critical recession velocity $\beta_{c r}=\varphi$ as phase criticality at cosmic scale [9].

Now we want to study this function in more detail, replacing the variable $p_{\tau}$ by $x$ and write the function $P$ as

$$
f(x)=x^{2} \frac{1-x}{1+x}
$$

Integration of $f(x)$ leads to

$$
\frac{1}{2} \int_{0}^{1} f(x) \mathrm{d} x=\ln (2)-\frac{2}{3}=0.026480514 \cdots \approx \frac{1}{12 \pi}
$$

The first derivative of $f(x)$ resulted in

$$
f^{\prime}(x)=\frac{\mathrm{d} f(x)}{\mathrm{d} x}=\frac{2 x\left(1-x-x^{2}\right)}{(x+1)^{2}}
$$

By comparing the nominator term $-\left(x^{2}+x-1\right)$ with Equation (11) the reference to the golden ratio becomes evident. The remaining multiplicative term caused a discontinuity at $x=-1$.

Higher derivatives of $f(x)$ lead to the following results

$$
\begin{gathered}
f^{\prime \prime}(x)=\frac{2\left(1-x^{3}-3 x^{2}-3 x\right)}{(x+1)^{3}} \\
f^{\prime \prime \prime}(x)=-\frac{12}{(x+1)^{4}}
\end{gathered}
$$

Special values for $f(x), f^{\prime}(x)$ and higher derivatives were summarized in the following Table 1.

If one connects the original function with energy, then its third derivative should be somewhat like a pressure. More surprisingly, the third derivative comes along as a very simple function with an origin value of -12 at $x=0$ and a value of $-12 \varphi^{4}$ at $x=\varphi$, underscoring the magic importance of number 12, found for instance as the number of vertices of the Platonic solid icosahedron. 


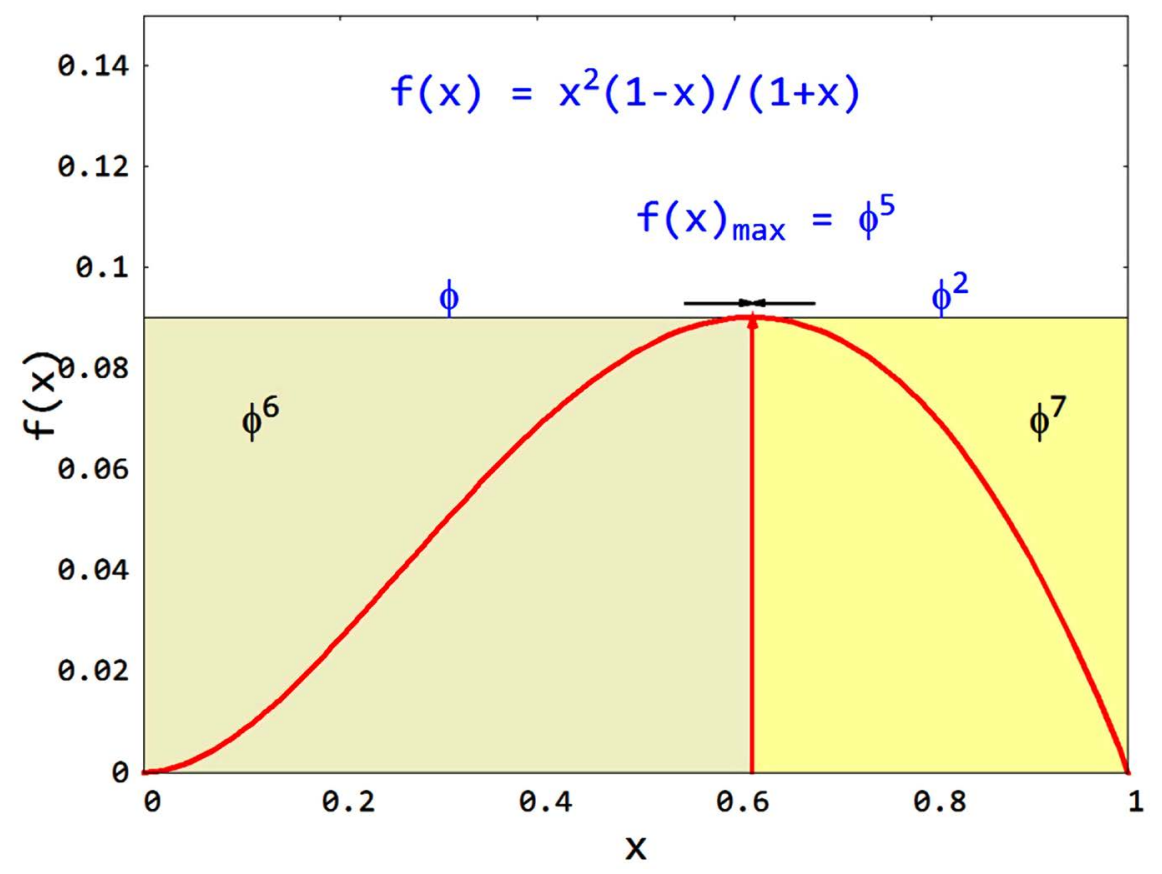

Figure 3. Hardy's quantum probability $P=f(x)$ for two particles [14] respectively $S u$ leiman's matter energy density [9] versus the entanglement variable $X$ (or recession velocity by Suleiman).

Table 1. Special values for the functions $f(x)$ and derivatives.

\begin{tabular}{ccccc}
\hline $\boldsymbol{x}$ & $f(x)$ & $f^{\prime}(x)$ & $f^{\prime \prime}(x)$ & $-f^{\prime \prime \prime}(x) / 12$ \\
\hline 0 & 0 & 0 & 2 & 1 \\
$1 / 3$ & $5 / 90=1 / 18$ & $5 / 24=0.208333$ & $-5 / 16=-0.31250$ & $(3 / 4)^{4}$ \\
$1 / 2$ & $1 / 12$ & $1 / 9$ & $-22 / 27=-0.8148148$ & $(2 / 3)^{4}$ \\
$\varphi$ & $\varphi^{5}$ & 0 & $-\left(1+\varphi^{6}\right)$ & $\varphi^{4}$ \\
1 & 0 & $-1 / 2$ & $-3 / 2$ & $(1 / 2)^{4}$ \\
\hline
\end{tabular}

\section{Superconductivity}

However, nature presents much more relationships to keep in mind, where the golden mean or its fifth power is involved, and superconductivity is no exception. So we must reassess the theory considering the dark matter surrounding the moving electrons, which dive into the dark after marriage, or in other words, become superconducting under special conditions. Recently, the present author suggested linking the optimum hole doping $\sigma_{0}$ of high- $T_{c}$ superconductors with the golden mean again in the form of its fifth power [17] [18] [19]

$$
\sigma_{0} \approx \frac{8}{\pi} \varphi^{5}=0.2293
$$

It was suggested recently that the same is true for conventional superconductors [18]. Obviously, this optimum is confirmed again near a quantum critical point in the superconductor phase diagram. In addition, the relation of the Fermi speed to the Klitzing speed comes out as [17] 


$$
\frac{v_{F}}{v_{K}} \approx \frac{2}{\pi} \varphi^{5}=0.0571
$$

Both relations document the fractal nature of the electronic response in superconductors. Also Prester had reported before about evidence of a fractal dissipative regime in high- $T_{c}$ superconductors [32].

In Figure 4 earlier results [17] that illustrate the dependence between the critical temperature and the mean cationic charge $\left\langle q_{c}\right\rangle$ for unconventional superconductors were completed with data points for the lanthanum and yttrium super-hydrides respectively $\mathrm{H}_{3} \mathrm{~S}$, showing the branch of $n$-type superconductors besides the $p$-type branch.

The prospect of developing a resilient theory of superconductivity is promised. Quantum entanglement of two moving electrons is influenced by local interaction of their interwoven dark matter surroundings, quoting the cogwheel picture of Suleiman [9]. In a recent contribution the present author designed the picture of two stretched electrons that locally interact to become superconducting. Such particle stretching into strands may in the end lead to a double-helically wounded wavy entity, which escapes in the dark.

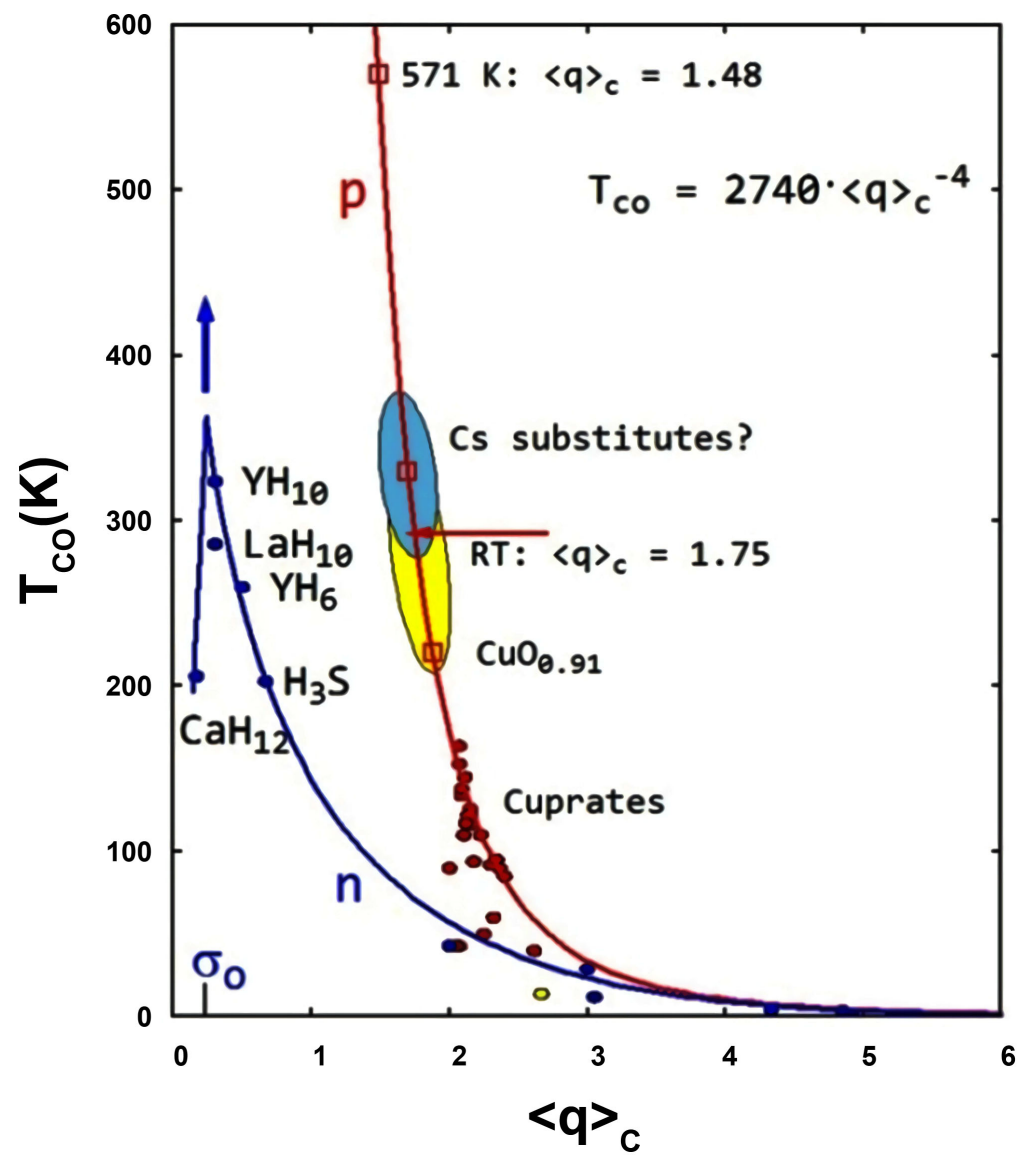

Figure 4. Mean cationic charge versus critical temperature $T_{c o}(\mathrm{~K})$ of superconducting compounds. $p$ : $p$-type branch, n: $n$-type branches having $\sigma<\sigma_{o}$ and $\sigma>\sigma_{o}$ ( $\sigma$ equivalent to $\left.\left\langle q_{c}\right\rangle\right)$. For more details see [17]. 


\section{Tammes Problem of Decorating a Unit Sphere with Hard Circles}

In 1930 the Dutch biologist Tammes [20] posed the question about the number and the arrangement of exit points in pollen grains. Reformulating the problem leads to the question what is the largest diameter of $N$ non-overlapping equal circles placed on the surface of a unit sphere, referred to as hard-spheres problem [33] [34] [35]. A sphere can't be tiled by using only hexagons. However, a perturbation of some hexagons in the hexagonal lattice into pentagons enables such a fit on the sphere, exemplified by the soccer ball structure [35]. In the following the energy of interaction is considered resulting from the arrangements of $N$ points on the surface of a unit sphere $S^{2}:=\left\{x \in \mathbf{R}^{3}:|x|=1\right\}$. The interaction is given by the power low potential $V=|x-y|^{\alpha},-2<\alpha<2$ of the Euclidian distance between two points $x, y \in S^{2}$. For $\alpha=0$ the potential $V=\ln \left(\frac{1}{|x-y|}\right)$ is used. Given a fixed configuration of $N$ points $\omega_{N}=\left(x_{1}, x_{2}, \cdots, x_{N}\right)$, the $\alpha$-energy sums up as [33] [36]

$$
E\left(\alpha, \omega_{N}\right):= \begin{cases}\sum_{1 \leq i<j \leq N} \ln \frac{1}{\left|x_{i}-x_{j}\right|}, & \text { if } \alpha=0 \\ \sum_{1 \leq i<j \leq N}\left|x_{i}-x_{j}\right|^{\alpha}, & \text { if } \alpha \neq 0\end{cases}
$$

Of importance is the determination of extremal energies for $N$ points on the sphere [34] [36]

$$
\varepsilon(\alpha, N):= \begin{cases}\inf _{\omega_{N} \in S^{2}} E\left(\alpha, \omega_{N}\right), & \text { if } \alpha \leq 0 \\ \sup _{\omega_{N} \in S^{2}} E\left(\alpha, \omega_{N}\right), & \text { if } \alpha>0\end{cases}
$$

Our concern should now be the special case $\varepsilon(0, N)$ for which a conjecture already exists [34] [36]

$$
\begin{gathered}
\varepsilon(0, N) \geq-\frac{1}{4} \ln \left(\frac{4}{e}\right) N^{2}-\frac{1}{4} N \ln N+O(N) \\
\lim _{N \rightarrow \infty} \frac{E(0, N)-\left(-\frac{1}{4} \ln \left(\frac{4}{e}\right) N^{2}-\frac{1}{4} N \ln N\right)}{N}=\mu
\end{gathered}
$$

with an estimate of $\mu=-0.026422 \ldots$ [36]. The bound $\mu$ may be compared with the result given for the integral over the matter energy density (or even the Hardy function) according to Equation (35). We assume that this last value should be the correct bound

$$
\mu=\frac{2}{3}-\ln (2)=-0.0264805 \cdots
$$

Quoting Saff et al. [37] respectively Rakhmanov et al. [36], numerical calculations indicated that logarithmic equilibrium points $(\alpha=0)$ with a generalized spiral point set $\hat{\omega}_{N}$ have the tendency to distribute themselves over a nearly regular spherical hexagonal net giving again a connection with $\mu$ (our interpretation) 


$$
E\left(0, \hat{\omega}_{N}\right)-\varepsilon(0, N) \leq 114 \cdot \ln N \approx \frac{3}{|\mu|} \ln N
$$

The interesting connection between minimal discrete energy on the sphere and matter energy density of the cosmos according to the information relativity theory [9] may suggest far-reaching consequences and should therefore be investigated more detailed in future.

\section{Viral Morphology and Beyond}

Nowadays we are faced with the worldwide pandemic spread of a very mobile corona virus. Therefore, the viral self-assembly and reproduction as a thermodynamic process comes ones again into focus. Viral self-assembly is intimately related to the before mentioned Tammes problem [20] of covering the surface of a sphere by optimally packed $N$ circular disks with the alteration that in case of viruses the coverage may be conformational-optimized by disks-like protein capsomers (subunits) having two different radii. Some time ago, Bruinsma et al. [38] showed that such a two-radius model as a form of structural free energy minimization prefers the icosahedral symmetry as well as the transition of sphere-like viruses to rod-like ones such as found for bucky-tube aggregates.

Models for the viral spread are quite complex, but may be likewise considered as noise dynamical phase transition with a critical point [39]. Here the golden number may come into play, which had dominated the development of first selfassembling precursors of life as well as later life itself and the ability to process and compress information with extreme speed.

Heretically put, the deadly virus acts as a corrective to human stupidity by clipping human dominance and restlessness for the good of the environment. Optimistically, the mankind, if it survives at all, could be able in the distant future to control respectively destroy any small pathogenic bodies via teleported information, influencing the dark matter halo of the particles.

In anticipation of the explanations in the next chapter, we may ask whether quantum computation with (stable) viral structures could be possible in future or by use of artificial microtubules build from helically rolled up hexagon lattice entities similar to tubulin proteins that then may operate under ambient conditions. The author recommends reading the seminal contributions of Penrose and Hameroff [40] [41] and the recently published remarkable contribution of Olson et al. [2] explaining in more detail parts of this topic. Tubulin protein molecules form linear chains which are able to self-assemble into $2 D$ sheets that can roll up to microtubules (Figure 5) [42].

The concept of a special arrangement of microtubules into a "heavy" hexagonal lattice with numbers that follow the Fibonacci number sequence and with vertices that minimize frequency collisions to protect the system from de-coherence, has been effectively refined again and again in nature by evolution. Not surprisingly, it resembles somewhat the qubit architecture of IBMs last quantum computer ( $Q C$ ) [43]. As in buckytubes, ballistic electron transport through the microtubules should be an essential property. Consequently, particles moving with 


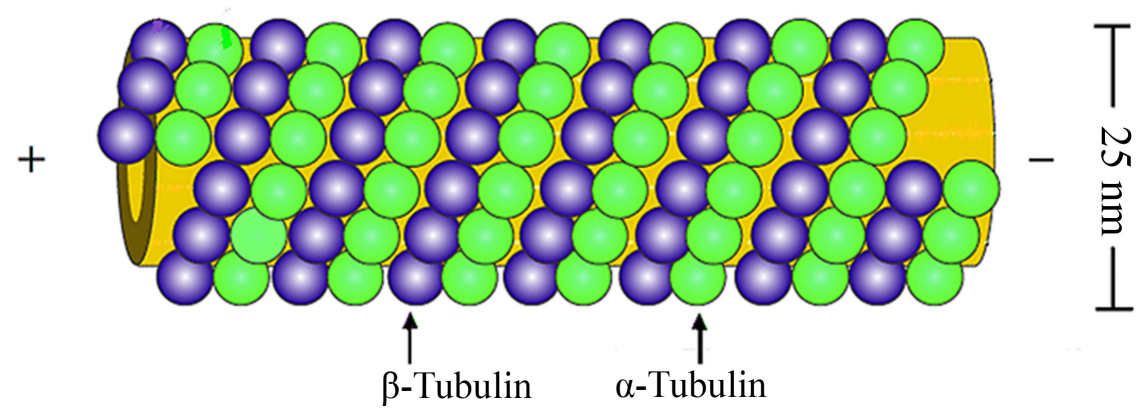

Figure 5. Sketch of a microtubule of helically rolled up tubulin protein subunit chains.

ballistic speed are entangled through their pronounced dark matter halo with the environment and can by that pass on and store information. This is an important result of the IRT of Suleiman [9].

\section{Quantum Computation with the Reverse Fibonacci Sequence and Fibonacci Anyons}

The golden mean $\varphi$ as the most irrational number found in nature with the simplest continued fraction representation at all is approximated by the division of two consecutive numbers of the Fibonacci number sequence [44] [45]. However, Janičko [23] recently introduced a new number sequence that he named reverse Fibonacci or Janickko sequence. The reverse sequence may be represented by the recursive formula beginning with the first two numbers as 0 and 1

$$
J_{n+2}=8\left(J_{n+1}-J_{n}\right)
$$

Comparable to the quadratic Equation (7), where solutions lead to the golden mean, we can formulate a quadratic equation for the reverse sequence, too. It yields

$$
J^{2}-8 J+8=0
$$

giving fundamental number $j_{1,2}$ as its two solutions [23]

$$
\begin{gathered}
j_{1}=4+\sqrt{8}=2(2+\sqrt{2})=6.82842712475 \cdots \\
j_{2}=4-\sqrt{8}=2(2-\sqrt{2})=1.171572875 \cdots
\end{gathered}
$$

As in the case of the golden mean also the reverse number $j_{1}^{-1}$ seems to be important

$$
j_{1}^{-1}=1-j_{2}^{-1}=\frac{j_{2}}{8}=0.1464466094067 \cdots
$$

Again, both numbers can be approximated by the ratio of consecutive Janičko numbers [23].

This number sequence may be important in calculations with quantum bits. The particle-wave duality is essential in the quantum information theory, where the unit of information is given by the quantum bit (qubit) coined by Schumacher [46], which exhibits the aspects of particle localization (counting) and wave interference to represent a signal with high fidelity [46]. Such two-state quantum system can be represented by the superposition principle: 
$S U(2) \cong H_{1}$ a $Q=\alpha|0\rangle+\beta|1\rangle$, where $\alpha$ and $\beta$ are complex numbers, where $\alpha^{2}+\beta^{2}=1$ [46]. A single qubit state can also be written as

$$
|\psi\rangle=\sqrt{p r}|0\rangle+\mathrm{e}^{i \phi} \sqrt{1-p r}|1\rangle
$$

where $0 \leq p r \leq 1$ is the probability of the bit being in the 0 state, and $0 \leq \phi \leq 2 \pi$ is the phase of the quantum. All possible values of $p r$ and the phase $\phi$ are reachable by application of quantum gates, realized by reliable and repeatable Josephson junctions. For instance, operating the quantum logical $T$-gate as quantum circuit with a phase of $\pi / 4$ that is mapped by the unitary matrix

$$
T=\left(\begin{array}{cc}
1 & 0 \\
0 & \mathrm{e}^{i \pi / 4}
\end{array}\right)
$$

one gets for $\operatorname{pr}(0)=j_{2}^{-1}=0.8535533 \cdots$ respectively for $\operatorname{pr}(1)=j_{1}^{-1}=0.1464466 \cdots$. In this way the connection of a single qubit with Janičko numbers is demonstrated as before suggested by Janičko itself [23]. The number $j_{1}^{-1}$ is near the fourth power of $\varphi: \varphi^{4}=0.14589803 \cdots$ and $j_{2} \approx \sqrt{2-\varphi}=1.17557050 \cdots$.

In contrast, topological quantum computation using Fibonacci anyons as qubits [47] [48] may be the ultimate approach because it naturally simulates processes which determine the speed and storage capacity of human mind. Fibonacci anyons have been proposed as two-dimensional quasi-particles exhibiting fractional quantum Hall states at 5/2 respectively $12 / 5$ filling fractions. As an example, a qubit can be build up from four Fibonacci anyons, where the fourth "inert" anyon may serve for error correction. The world lines of such anyons pass around one another to form braids. Braids can be thought of as quantum circuits forming the logic gates, which building up the computer [49]. Their non-Abelian braiding statistics with only two particle types are shown to be suitable for quantum computation. The two particle types or topological charges are the trivial type 1 and the non-trivial type $\tau$, which are self-dual and being their own antiparticles $\left(1=\mathbf{1}^{*}, \tau=\tau^{*}\right)$. The combination or fusion of anyons governed by fusion rules may be thought of as a measurement, graphically displayed by a fusion tree [50]. The probability of a fusion is denoted as quantum dimension $d_{i}$. The quantum dimension of $\tau$ is $\varphi^{-1}$, the inverse of the golden mean. When two $\tau$ anyons are combined, the probability pr to yield 1 is $p r_{0}=\varphi^{2}$, and to yield $\tau$ is $p r_{1}=\varphi \quad[50]$.

For a multi-anyon system the number of fusion paths resulted exactly in the Fibonacci number, and the move accompanied by the consecutive fuse of the anyons from left to right can be represented by a $F$ matrix. For more than three anyons consistency equations for the fusion trees in form of pentagon relations deliver the entries of the $F$-matrices [50]. Up to arbitrary phases the $2 \times 2$ unitary $F$ matrix reads

$$
F_{\tau}^{\tau \tau \tau}=\left(\begin{array}{ll}
F_{11} & F_{1 \tau} \\
F_{\tau 1} & F_{\tau \tau}
\end{array}\right)=\left(\begin{array}{cc}
\varphi & \sqrt{\varphi} \\
\sqrt{\varphi} & -\varphi
\end{array}\right)
$$

where again $\varphi$ is the golden number (Figure 6). 


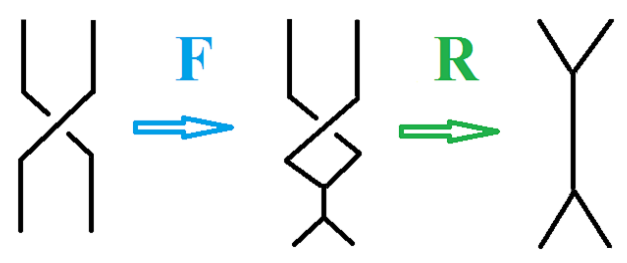

Figure 6. Removing crossings by $F$ and $R$ moves after Slingerland [51].

The braiding operator $B$ can be derived by matrix multiplication of $F$-matrices with $R$-matrices. When the $R$-matrix is given by diagonal spin factors

$$
R=\operatorname{diag}\left(\mathrm{e}^{\frac{4 \pi i}{5}}, \mathrm{e}^{-\frac{3 \pi i}{5}}, \mathrm{e}^{-\frac{3 \pi i}{5}}, \mathrm{e}^{\frac{4 \pi i}{5}}, \mathrm{e}^{-\frac{3 \pi i}{5}}\right)
$$

and the extended $5 \times 5 F$-matrix by

$$
F=\left(\begin{array}{ccccc}
1 & & & & \\
& 1 & & & \\
& 1 & & \\
& & \varphi & \sqrt{\varphi} \\
& & \sqrt{\varphi} & -\varphi
\end{array}\right)
$$

then one obtains finally the $B$ matrix as [50]

$$
B=F^{-1} R F=\left(\begin{array}{cccc}
\mathrm{e}^{4 \pi i / 5} & & & \\
& \mathrm{e}^{-3 \pi i / 5} & & \\
& \mathrm{e}^{-3 \pi i / 5} & & \\
& & \varphi \mathrm{e}^{-4 \pi i / 5} & -\sqrt{\varphi} \mathrm{e}^{-2 \pi i / 5} \\
& & -\sqrt{\varphi} \mathrm{e}^{-2 \pi i / 5} & -\varphi
\end{array}\right)
$$

"Pentagon" moves respectively "hexagon" ones are third order polynomial equations in many variables with much more equations than variables, but not always existing solutions.

Freedman, Larsen and Wang [49] confirmed the universal applicability of the following approximated quantity $p r$ (probability) for quantum computation

$$
p r=\left|\frac{V(L, t)}{\left(t^{\frac{1}{2}}+t^{-\frac{1}{2}}\right)^{g-1}}\right|^{2}
$$

where $V(L, t)$ is the Jones polynomial of a link $L[52], t=\exp \left(\frac{2 \pi i}{r}\right)$ is a principal root of unity with $r=5$ or $r \geq 7$, and $g$ is the bridge number ${ }^{1}$. Lattice roots of unity, expressed in the parlance of a crystallographer, with $r \in\{1,2,3,4,6\}$ are excluded. Field and Simula [53] reported recently about the connection between Fibonacci anyons and the Jones polynomial at the fifth root of unity $t=\exp \left(\frac{2 \pi i}{5}\right)$.

${ }^{1}$ The bridge number $g=2 n$ is the number of $U$-turns at each end of a capped braid (plat diagram). 
The magnitude of the Jones polynomial [47] [54] of the Hopf link $^{2}$ is related to the probability $\operatorname{pr}\left(|0\rangle_{5}\right)$ simply by [53]

$$
V_{\text {Hopf }}\left(\mathrm{e}^{\frac{2 \pi i}{5}}\right)=\sqrt{p r\left(|0\rangle_{5}\right)} \cdot \varphi^{-1}=\varphi
$$

with only two elementary physical braiding operations used before measuring the annihilation probability of the anyons upon fusion [53].

This short survey may serve to demonstrate the importance of the golden number for the development of quantum computation that should stimulate research in a direction along which way nature has always evolutionary and subtly worked. In this sense the seminal ideas of Penrose and Hameroff [40] [41] should be further elaborated (see Section 7). In the next chapter new golden-mean-based quantum computer architecture was proposed copying nature's most effective principles.

\section{Proposal of Quantum Computer Architecture Based on Fibonacci Net Microtubules}

Besides the compounds currently applied such as GaAs hetero-structures or graphene, which show the fractional quantum Hall effect ( $F Q H E$ ), the investigation of other inorganic (semiconducting) compounds is recommended, for instance ferroelastic compounds or compounds that show Fibonacci-like unit-cell decoration [55] [56]. Interfacial twin walls act as sink for charge carriers or defects generating locally superconductivity or ferroelectricity as striking properties [57]. Fibonacci unit-cell tailoring was displayed in Figure 7. Geometric relations ( Fibonacci relations) between these cells can be followed by studying [55] [56] respectively in the Appendix. The small black hexagonal cell (sub-cell) is rotated by an angle of $\alpha=\operatorname{atan}\left(\frac{5}{3 \sqrt{3}}\right)-30^{\circ}=13.898^{\circ}$ to generate the blue outlined unit-cell with lattice parameter $a$ that has 13 times the volume of the small cell. One may repeat this procedure to obtain a super-cell and so on. Furthermore, it should be possible to roll up this $2 D$ net into a microtubule (Figure 8 ) and plug tubules with different radii or orientation into each other to produce a multi-shell structure. The starting small cell is then helically wound at the twist angle $\alpha$. It needs 13-times the small cell lattice parameter $a_{s u b}$ to reach again identity with a large cell lattice point. In this way the diameter of the smallest geometrically possible microtubule is calculated to be $d_{1} \approx 13 a_{\text {sub }} \cdot \cos a_{\text {sub }} \cdot \cos (\alpha) / \pi=3.5 \cdot a / \pi$. Interesting is $d_{6}=21 \cdot \frac{a}{\pi}$ showing a further Fibonacci number. By a full turn one gains a height in filament direction of $h_{1}=\frac{\sqrt{39}}{2} a_{\text {sub }} \approx \pi \cdot a_{\text {sub }}$. If you look through the microtubule perpendicular to the filament direction, you will see the mirror image on the back somewhat offset.

${ }^{2}$ One speaks of a Hopflink, representing a $(2,2)$-torus link, when two circles are linked together with a crossing number of two. 

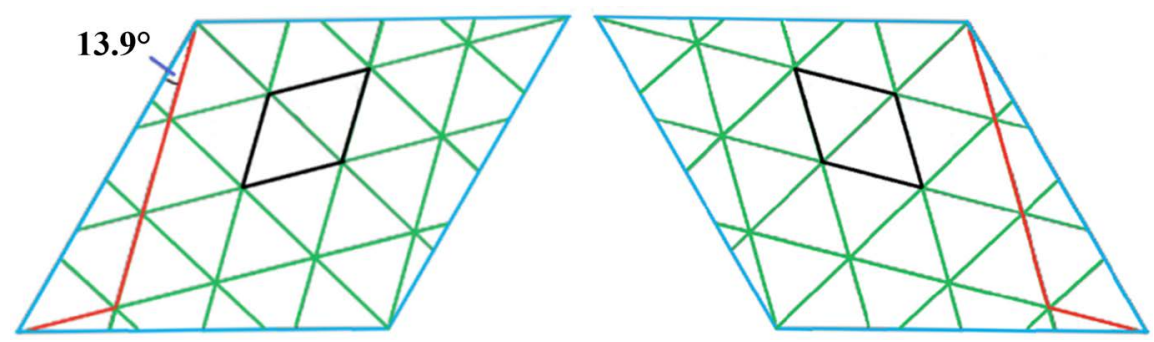

Figure 7. Fibonacci arrangement of a hexagonal net and its mirror image [55] [56]. The light-blue outlined unit-cell contains 13 sub-cells, offset by an angle of $\alpha=13.9^{\circ}$.

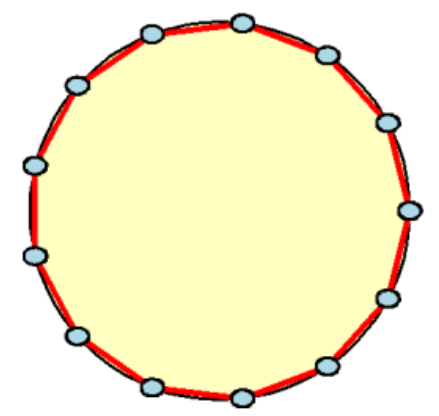

Figure 8. Helically twisted microtubule projected down the filament direction with 13 light-blue atoms or atom groups on sub-lattice positions.

In practice it may be a sophisticated task to produce such a tubule, but nowadays it should be possible. The future will show whether such a composite is applicable for $Q C$, after the right compound with optimal physical properties is found. One could start with doped graphene rolled up in the explained Fibonacci twisting. The Kitaev honeycomb lattice approach may be applied in an extended form to this special lattice variant [58].

Interestingly, the helically twisted tubulin microtubules show strongest reinforcement of the ordered pattern when the protofilament number $n$ equals 13 [59] [60]. This supports the proposal for a twisted Fibonnaci net as displayed in Figure 7.

A very stimulating question is what happens if the microtubule is compressed or dilated due to vibration in filament direction finally going through an incommensurability state represented by $13+\varphi^{5}=\left(2+\varphi^{-1}\right)^{2}=13.0901699 \cdots$ instead of 13 twist steps (see Appendix). One may tentatively characterize such a behavior also by a phase transition. A highly effective quantum computer could possibly be realized based on such a principle. Besides the explained longitudinal adaption of the twisted net, its sense of rotation, optimal diameter, and the freedom given by the decoration of the net itself, there are many more degrees of freedom, if one constructs a hexagonal lattice of such 13-step microtubules with non-hexagonal internal symmetry. Neighboring filament can be rotated one again the other violating the hexagonal symmetry. In addition, the border of the assembled filaments may be important. This is what nature has already optimized to equip its creatures with perception, memory and intelligence. 


\section{Conclusions}

This contribution reviewed and extended results of different scientific disciplines that involve the golden mean $\varphi$ as very important number of nature. It covered especially phase transitions governed by the fifth power of $\varphi$, found in Baxter's hard lattice approaches of statistical mechanics as well as in energy density relations of the cosmos, comparing results of the $\varepsilon$-infinity theory of El Naschie with the conclusive findings of Suleiman's scale-free information relativity theory (IRT) besides Hardy's quantum entanglement probability. Also superconductivity is described as an inherently fractal property connected with the interaction of two moving electrons entangled and finally paired by way of their dark wavy surroundings. Since Klein's research on the icosahedron many others solved and combined fundamental scientific questions beyond icosahedral structures. From Tammes problem of decorating a unit sphere with hard circles one may be guided to viral morphology as well as phase transition, self-assembly and reproduction of viruses. Are there connections between the energy density of the cosmos with Tammes problem?

The golden mean is involved in the development of modern topological quantum computation, too. The performance of topological quantum computation with Fibonacci anyons can give a vague idea of how the human brain actually processes and stores information. It may be highly interesting to develop artificial self-assembling structures based for instance on the tubulin protein or viruses that can form helically wounded microtubules with the ability to store and to process information. A proposal was made for a twisted microtubule tailors from a rolled up Fibonacci net. Quantum computation is easier understandable, if one accepts the suggestions of Suleiman that quantum entanglement is a local realistic phenomenon "where each moving particle will be permeated by the dark matter halo of the other one making the two physically entangled" [15]. Essential suggestions of Irvin and scientific fellows about the golden ratio as a fundamental constant of nature could be ennobled by considering the IRT theory [8] [61]. The author wants to encourage "down-to-earth" physicists to follow and complete these suggestions and the perspective views of the present author, including the proposed change of some important physical constants [19] [62].

\section{Acknowledgements}

The author appreciates valuable suggestions given by a competent and cooperative reviewer.

\section{Conflicts of Interest}

The author declares no conflicts of interest regarding the publication of this paper.

\section{References}

[1] Olson, S. (2006) The Golden Section: Nature's Greatest Secret. Bloomsbury, London, $64 \mathrm{p}$. 
[2] Olsen, S., Marek-Crnjak, L., He, J.H. and El Naschie, M.S. (2020) A Grand Unification of the Sciences, Art \& Consciousness: Rediscovering the Pythagorean Plato's Golden Mean Number System. Journal of Progressive Research in Mathematics, 16, 2880-2931.

[3] Sherbon, M.A. (2014) Fundamental Nature of the Fine-Structure Constant. International Journal of Physical Research, 2, 1-9. https://doi.org/10.14419/ijpr.v2i1.1817

[4] Otto, H.H. (2017) Continued Fraction Representations of Universal Numbers and Approximations. Researchgate.net, 1-4.

[5] El Naschie, M.S. (2013) Quantum Entanglement: Where Dark Energy and Negative Gravity plus Accelerated Expansion of the Universe Comes from. Journal of Quantum Information Science, 3, 57-77. https://doi.org/10.4236/jqis.2013.32011

[6] Ho, M.-W. (2014) Golden Geometry and E-Infinity Fractal Spacetime. Story of Phi Part 5. Researchgate.

[7] Marek-Crnjac, L. (2013) Cantorian Space-Time Theory. Lambert Academic Publishing, Saarbrücken, 1-50.

[8] Irwin, K. (2019) Towards the Unification of Physics and Number Theory. Reports in Advances of Physical Sciences 3, Article ID: 1950003.

[9] Suleiman, R. (2019) Relativizing Newton. Nova Scientific Publisher, New York, $1-207$.

[10] Andrews, G.E. (1981) Ramanujan's “Lost” Notebook. III. The Rogers-Ramanujan Continued Fraction. Advances in Mathematics, 41, 186-208. https://doi.org/10.1016/0001-8708(81)90015-3

[11] Baxter, R.J. (1980) Hard Hexagons: Exact Solution. Journal of Physics A: Mathematical and General, 13, L61-L70. https://doi.org/10.1088/0305-4470/13/3/007

[12] Baxter, R.J., Enting, I.G. and Tsang, S.K. (1980) Hard-Square Lattice Gas. Journal of Statistical Physics, 22, 465-489. https://doi.org/10.1007/BF01012867

[13] Baxter, R.J. (1982) Exactly Solved Models in Statistical Mechanics. Academic Press, London.

[14] Hardy, L. (1993) Nonlocality for Two Particles without Inequalities for Almost All Entangled States. Physical Review Letters, 71, 1665-1668. https://doi.org/10.1103/PhysRevLett.71.1665

[15] Mermin, N.D. (1994) Quantum Mysteries Refined. American Journal of Physics, 62, 880-887. https://doi.org/10.1119/1.17733

[16] Otto, H.H. (2018) Reciprocity Relation between the Mass Constituents of the Universe and Hardy's Quantum Entanglement Probability. World Journal of Condensed Matter Physics, 8, 30-35. https://doi.org/10.4236/wjcmp.2018.82003

[17] Otto, H.H. (2016) A Different Approach to High- $\mathrm{T}_{c}$ Superconductivity: Indication of Filamentary-Chaotic Conductance and Possible Routes to Superconductivity above Room Temperature. World Journal of Condensed Matter Physics, 6, 244-260. https://doi.org/10.4236/wjcmp.2016.63023

[18] Otto, H.H. (2019) Super-Hydrides of Lanthanum and Yttrium: On Optimal Conditions for Achieving near Room Temperature Superconductivity. World Journal of Condensed Matter Physics, 9, 22-36. https://doi.org/10.4236/wjcmp.2019.91002

[19] Otto, H.H. (2020) Reciprocity as an Ever-Present Dual Property of Everything. Journal of Modern Physics, 11, 98-121. https://doi.org/10.4236/jmp.2020.111007

[20] Tammes, P.M.L. (1930) On the Origin of Number and Arrangement of the Places of Exit on Pollen Grains. Dissertation, J.H. De Bussy, Groningen. (See also Recueil des Travaux Botanique Néerlandais, 27, 1-84) 
[21] Trebst, S., Troyer, M., Wang, Z., Ludwig, A.W.W. (2009) A Short Introduction to Fibonacci Anyon Models. arXiv: 0902.3275v1 [cond-mat.stat-mech], 1-24.

[22] Field, B. and Simula, T. (2018) Introduction to Topological Quantum Computation with Non-Abelian Anyons. arXiv: 1802.06176v2 [quant-ph].

[23] Janičko, O. (2018) New Fundamental Discovery of the Reverse Fibonacci Sequence. Academia.edu, 1-6.

[24] Onsager, L. (1944) Crystal Statistics. I. A Two-Dimensional Model with Order-Disorder Transition. Physical Review, 65, 117-149. https://doi.org/10.1103/PhysRev.65.117

[25] Finch, S.R. (2003) Mathematical Constants. Cambridge University Press, New York.

[26] Assis, M., Jacobsen, J.L., Jensen, I., Maillard, J.M. and McCoy, B.M. (2013) The Hard Hexagon Partition Function for Complex Fugacity. Journal of Physics A: Mathematical and Theoretical, 46, Article ID: 445202. https://doi.org/10.1088/1751-8113/46/44/445202

[27] Tracy, C.A., Grove, L. and Newman, M.F. (1987) Modular Properties of the Hard Hexagon Model. Journal of Statistical Physics, 48, 477-502. https://doi.org/10.1007/BF01019683

[28] Klein, F. (1884) Vorlesungen über das Ikosaeder und die Auflösung der Gleichungen vom fünften Grad. Verlag B. G. Teubner, Leipzig.

[29] Coldea, R., Tennant, D.A., Wheeler, E.M., Wawrzynska, E., Prabhakaram, D., Telling, M., Habicht, K., Smeibidl, P. and Kiefer, K. (2010) Quantum Criticality in an Ising Chain: Experimental Evidence for Emergent E8 Symmetry. Science, 327, 177-180. https://doi.org/10.1126/science.1180085

[30] Fendley, P., Sengupta, K. and Sanchev, S. (2004) Competing Density-Wave Orders in a One-Dimensional Hard-Boson Model. Physical Review B, 69, Article ID: 075106. https://doi.org/10.1103/PhysRevB.69.075106

[31] Cantor, G. (1932) Gesammelte Abhandlungen. Mathematischen und Philosophischen Inhalts. Springer, Berlin. https://doi.org/10.1007/978-3-662-00274-2

[32] Prester, M. (1999) Experimental Evidence of a Fractal Dissipative Regime in High- $\mathrm{T}_{\mathrm{c}}$ Superconductors. Physical Review B, 60, 3100-3103.

https://doi.org/10.1103/PhysRevB.60.3100

[33] Wagner, W. (1990) On Means of Distances on the Surface of a Sphere (Lower Bounds). Pacific Journal of Mathematics, 114, 389-398. https://doi.org/10.2140/pjm.1990.144.389

[34] Zhou, Y.M. (1995) Arrangements of Points on the Sphere. Ph.D. Thesis, University of South Florida, Tampa, FL.

[35] Aste, T. and Weaire, D. (2000) The Pursuit of Perfect Packing. Taylor and Francis Group, Abingdon-on-Thames, 108-110.

[36] Rakhmanov, E.A., Saff, E.B. and Zhou, Y.M. (1994) Minimal Discrete Energy on the Sphere. Mathematical Research Letters, 1, 647-662. https://doi.org/10.4310/MRL.1994.v1.n6.a3

[37] Saff, E.B. and Kuijlaars, A.B.J. (1997) Distributing Many Points on a Sphere. The Mathematical Intelligencer, 19, 5-11. https://doi.org/10.1007/BF03024331

[38] Bruinsma, R.F., Gelhart, W.M., Reguera, D., Rudnick, J. and Zandi, R. (2003) Viral Self-Assembly as a Thermodynamic Process. Physical Review Letters, 90, Article ID: 248101. https://doi.org/10.1103/PhysRevLett.90.248101

[39] Drake, J.M., Brett, T.S., Chen, S., Epureanu, B.I., Ferrari, M.J., Marty, E., Miller, P.B., O’Dea, E.B., O’Regan, S.M., Park, A.W. and Rohani, P. (2019) The Statistics of Epidemic Transitions. PLoS Computational Biology, 15, e1006917. https://doi.org/10.1371/journal.pcbi.1006917 
[40] Penrose, R. and Hamedoff, S.R. (1995) Quantum Computing in Microtubules: Self-Collapse as a Possible Mechanism for Consciousness. Toward a Science of Consciousness. MIT Press, Cambridge, MA.

[41] Penrose, R. and Hamedoff, S.R. (2011) Consciousness in the Universe: Neuroscience, Quantum Space-Time Geometry and Orch OR Theory. Journal of Cosmology, 14, 1-50.

[42] Sahu, S., Ghosh, S., Fujita, D. and Bandyopadhyay, A. (2014) Live Visualization of Single Isolated Tubulin Protein Self-Assembly via Tunneling Current Effect of Electromagnetic Pumping during Spontaneous Growth of Microtubule. Scientific Reports, 4, Article No. 7303. https://doi.org/10.1038/srep07303

[43] Chamberland, C., Zhu, G., Yoder, T.J., Hertzberg, J.B. and Cross, A.W. (2019) Topological and Subsystem Codes on Low-Degree Graphs with Flag Qubits. ArXiv: 1907.09528v2 [quant-ph].

[44] Pisano, L. (1202) Fibonacci's Liber Abaci (Book of Calculation). Biblioteca Nazionale Centrale di Firenze, Firenze.

[45] Lucas, E. (1891) Theorie des nombres. Gauthier-Villars, Paris.

[46] Schumacher, B. (1995) Quantum Coding. Physical Review A, 51, 2738-2747. https://doi.org/10.1103/PhysRevA.51.2738

[47] Aharonov, D., Jones, V. and Landau, Z. (2006) A Polynomial Quantum Algorithm for Approximating the Jones Polynomial. Proceedings of the 38th Annual ACM Symposium on Theory of Computing, Seattle WA, May 2006, 427-436. https://doi.org/10.1145/1132516.1132579

[48] Nayak, C., Simon, S.H., Stern, A., Freedman, M. and Das Sarma, S. (2008) Non-Abelian Anyons and Topological Quantum Computation. Reviews of Modern Physics, 80, 1083-1159. https://doi.org/10.1103/RevModPhys.80.1083

[49] Freedman, M.H., Larsen, M.J. and Wang, Z. (2002) The Two-Eigenvalue Problem and Density of Jones Representation of Braid Groups. Communications in Mathematical Physics, 228, 177-199. https://doi.org/10.1007/s002200200636

[50] Trebst, S., Troyer, M., Zhenghan, W. and Ludwig, A.W.W. (2009) A Short Introduction to Fibonacci Anyon Models. arXiv: 0902.3275v1 [cond-mat.stat-mech].

[51] Slingerland, J. (2007) Anyon Models-Theory, Interferometry, Bose Condensation. Presentation, UC Riverside, Caltech, California, USA.

[52] Jones, V.F.R. (1985) A Polynomial Invariant for Knots via Von Neumann Algebras. Bulletin (New Series) of the American Mathematical Society, 12, 103-111. https://doi.org/10.1090/S0273-0979-1985-15304-2

[53] Field, B. and Simula, T. (2018) Introduction to Topological Quantum Computation with Non-Abelian Anyons. Quantum Science and Technology, 3, Article ID: 045004. https://doi.org/10.1088/2058-9565/aacad2

[54] Kuperberg, G. (2015) How Hard It Is to Approximate the Jones Polynomial? Theory of Computing, 11, 183-219. https://doi.org/10.4086/toc.2015.v011a006

[55] Otto, H.H. (2015) Pyroelectric $\mathrm{Bi}_{5-\mathrm{x}}\left(\mathrm{Bi}_{2} \mathrm{~S}_{3}\right)_{39} \mathrm{I}_{12} \mathrm{~S}$ : Fibonacci Superstructure, Synthesis Options and Solar Cell Potential. World Journal of Condensed Matter Physics, 5, 66-77. https://doi.org/10.4236/wjcmp.2015.52010

[56] Otto, H.H. (1965) Zur Kristallchemie synthetischer Blei-Wismut-Spießglanze. Thesis, TU, Berlin.

[57] Salje, E. (2012) Ferroelastic Materials. Annual Review of Materials Research, 42, 265-283. https://doi.org/10.1146/annurev-matsci-070511-155022 
[58] Kitaev, A. (2008) Anyons in an Exactly Solved Model and Beyond. arXiv: 0506438v3 [cond-mat.mes-hall], 1-113.

[59] Wade, R.H., Chrétien, D. and Job, D. (1990) Characterization of Microtubule Protofilament Numbers. How Does the Surface Lattice Accommodate? Journal of Molecular Biology, 212, 775-786. https://doi.org/10.1016/0022-2836(90)90236-F

[60] Ti, S.C., Alushin, G.M. and Kapoor, T.M. (2018) Human $\beta$-Tubulin Isotypes Can Regulate Microtubule Protofilament Number and Stability. Developmental Cell, 47, 175-190. https://doi.org/10.1016/j.devcel.2018.08.014

[61] Irvin, K., Amaral, M.M., Aschheim, R. and Fang, F. (2017) Quantum Walk on a Spin Network and the Golden Ratio as a Fundamental Constant of Nature.

[62] Otto, H.H. (2020) A Primer of Important Natural Numbers and Revisited Fundamental Physical Constants.

[63] Fang, F., Irvin, K., Kovacs, J. and Sadler, G. (2013) Cabinet of Curiosities: The Interesting Geometry of the Angle $\beta$. 


\section{Appendix}

Roots of unity: By solving the quartic polynomial $\phi_{5}(x)=\sum_{n=0}^{4} x^{n}$ one yields the four primitive roots of unity $\omega_{i} \quad(i=1$ to 4 ) here notably expressed by terms of the golden mean

$$
\begin{gathered}
\omega_{1,2}=\frac{\varphi}{2} \pm \frac{i}{2} \sqrt{1+\varphi^{-2}}=\frac{\varphi}{2} \pm \frac{i}{2} \sqrt{2+\varphi^{-1}} \\
\omega_{3,4}=-\frac{\varphi^{-1}}{2} \pm \frac{i}{2} \sqrt{1+\varphi^{2}}=-\frac{\varphi^{-1}}{2} \pm \frac{i}{2} \sqrt{2-\varphi}
\end{gathered}
$$

About the Fibonacci number 13: This number is very special because its square root, coming along as the unit-cell-sub-cell parameter ratio of the $\mathrm{Fi}^{-}$ bonacci net approach, can be approximated by $2+\varphi^{-1}$

$$
\sqrt{13}=3.60555 \cdots \approx 2+\varphi^{-1}=3.6180339887 \cdots
$$

Furthermore, the exact adjustment connects the number 13 with the fifth power of $\varphi$, which number was the main concern of this contribution

$$
2+\varphi^{-1}=\sqrt{5} \varphi^{-1}=\sqrt{13+\varphi^{5}}=\sqrt{13.0901699 \cdots}=3.6180339887 \cdots
$$

When adding to the Hardy function [14] respectively Suleiman's matter energy density relation [9] an amount of 3 as a solely mathematical procedure, for the present without physical background, giving then the maximum of $5 \varphi$ instead of $\varphi^{5}$ at $x=\varphi$, the function is changed to

$$
f(x)=3+\frac{(1-x) x^{2}}{(1+x)}
$$

An exceptional result is obtained for the $x$ value at zero of this function

$$
x=\frac{5}{3}\left(\sqrt[3]{10^{2}}+\sqrt[3]{10^{1}}+\sqrt[3]{10^{0}}\right)=5 \times 2.59867450788 \cdots=12.993372 \cdots \approx 13
$$

Crystallography of the Fibonacci $2 D$-Lattice: The reciprocal sub-cell is put up according to [55] by the strongest hexagonal basis reflections $31 \overline{4} 0$ and $52 \overline{7} 0$. Their Miller-Bravais index relations represent Fibonacci numbers respectively products of them

$$
\begin{gathered}
(31 \overline{4} 0): h^{2}+k^{2}+h k=13 \\
(52 \overline{7} 0): h^{2}+k^{2}+h k=39=3 \times 13
\end{gathered}
$$

Also remarkable, the sine of the twist angle $\alpha=13.898^{\circ}$ can be approximated well by the ratio of $\pi$ and number $13+\varphi^{5}$

$$
\sin (13.898)=0.240192 \cdots \approx \frac{\pi}{13+\varphi^{5}}=0.239996 \cdots=\sin (13.886)
$$

The Fibonacci net can be generated by application of symmetry operations of the two-dimensional symmetry group p6. One needs three positions to get the 13 lattice points. Two general six-fold positions with coordinates in fractions of again 13 , besides the origin, yield the lattice points: $x_{1}=0, y_{1}=0 ; x_{2}=\frac{4}{13}, y_{2}=\frac{3}{13}$; $x_{3}=\frac{5}{13}, y_{3}=\frac{7}{13}$ (Figure 9). 


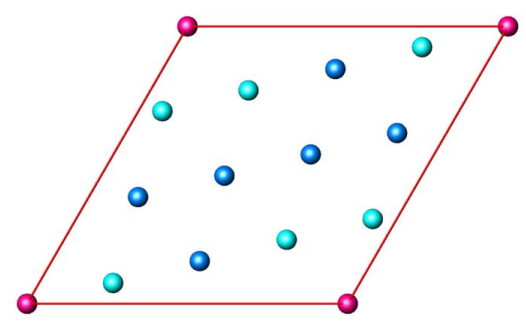

Figure 9. Illustration of the three differently colored atomic positions of the $2 D$ Fibonacci net.

Cabinet of curiosities: With this title Fang et al. [63] published a result concerning the offset angle of chained aggregates of tetrahedra with coincident faces given by the relation

$$
\beta=\arccos \left(\frac{3 \varphi^{-1}-1}{4}\right)=15.5224 \cdots
$$

However, one can approximate this angle very well by a relation that uses the fifth power of $\varphi$

$$
\beta \approx 3 \cdot \arcsin \left(\varphi^{5}\right)=15.52015 \cdots
$$

Geometric interpretation of $\varphi^{5}$ : The angle of $\beta / 3=5.173 \cdots$ is also near the difference of the half inner angles between pentagon and hexagon being $36^{\circ}-30^{\circ}=6^{\circ}$. The difference in the triangle areas between both regular polygons yielded a value near $\varphi^{5}=0.090169943 \cdots$

$$
\frac{A_{6}}{6}-\frac{A_{5}}{5}=\frac{a^{2}}{4}\left(\sqrt{3}-\sqrt{1+\frac{1}{\varphi+0.5}}\right)=0.088917221 \cdots a^{2}
$$

where $A_{6}$ and $A_{5}$ are the full areas of the hexagon respectively pentagon with edge length $a$. When the pentagon is only slightly rotated about the edge against the hexagon plane, and then the projected area is subtracted, one can well adapt the exact value of $\varphi^{5}$. 